\title{
Study on the Application of the Bowtie Methodology for the Assessment of Ergonomic Risks in the Industrial Field
}

\author{
Adrian ISPĂȘOIU \\ Transilvania University of Brasov, Romania, adrian.ispasoiu@unitbv.ro \\ Ioan MILOSAN \\ Transilvania University of Brasov, Romania, milosan@unitbv.ro \\ Ana Maria Florina ISPĂȘOIU \\ Maastricht University, Netherlands, ispasoiuana@yahoo.com \\ Cristina MEIȚĂ (GOGELESCU) \\ POLITEHNICA University of Bucharest, Romania, cristina.meita@bogart.ro
}

\begin{abstract}
Background and objective: Bowtie Analysis is an advanced risk assessment tool that allows users to view the risk map and illustrate the pathways from their causes to potential effects. In addition, the Bowtie analysis allows the assignment of controls for each identified cause and the application of key risk indicators, to help track the performance of risk controls. The aim of this research is to assess the risk of injury or damage to health for workers who are exposed to ergonomic risks, using the Bowtie methodology. Methods and materials: This paper presents an applied study of the Bowtie methodology, a proactive and systematic risk assessment technique, for the analysis and assessment of ergonomic risks in the industry. In this paper, a semi-quantitative method BTRA (Bowtie Risk Assessment) of estimating and evaluating the ergonomic risk was used, the method having the results of the Bowtie diagram as input data. The research analyzed the activity: welding metal parts, an activity associated with "danger" because it generates many risks of injury and damage to workers' health. Results: Analyzing the results of the assessment of the ergonomic risks generated by incorrect and/or uncomfortable positions during the activity of welding of the workpieces in the production hall, it is found that there is a high probability of occurrence of musculoskeletal disorders but also of serious injury to the worker caused in particular by the non-use of the appropriate means of working at height/semi-height and the use of improvisations, as well as due to the lack of mechanized means for gripping and rotating the workpiece in a position favorable to the worker. Conclusion: Analyzing the results obtained in this paper, it can be concluded the following most important findings, such as: The Bowtie methodology has proven its efficiency and effectiveness in identifying causes and effects in the event of occurrence of a Hazardous Event (Top Event) and the BTRA assessment method is easy to use in combination with the Bowtie method, taking into account the causes and consequences and assessing the effectiveness of proactive and reactive barriers, then classifying the criticality of causes and effects and finally estimating the probability and severity values based on which the risk is assessed.
\end{abstract}

\section{Keywords}

ergonomics, risk assessment, bowtie methodology, musculoskeletal disorders

\section{Introduction}

Ergonomic issues directly affect the safety and health of workers in all workplaces. In industries where activities with high risks of injury to workers are carried out, the basic rules on ergonomics are often not followed and this results in the adoption of actions with totally uncontrolled risks on the part of workers. The assessment of the risks of injury in such situations must be carried out systematically and rigorously. In this regard, an evaluation method has been developed based on the Bowtie method.

The Bowtie method is a risk assessment method which consists in building a qualitative diagram, which improves the understanding of risk and can be used in the analysis and presentation of how to conduct a high-risk scenario. The name of the method is given by the shape of the diagram that was created, which resembles a bow tie (Fig. 1). The essence of the Bowtie method is to analyze the various risk scenarios that unfold around a certain danger and the way in which the organization stops these scenarios from manifesting [1-3]. 


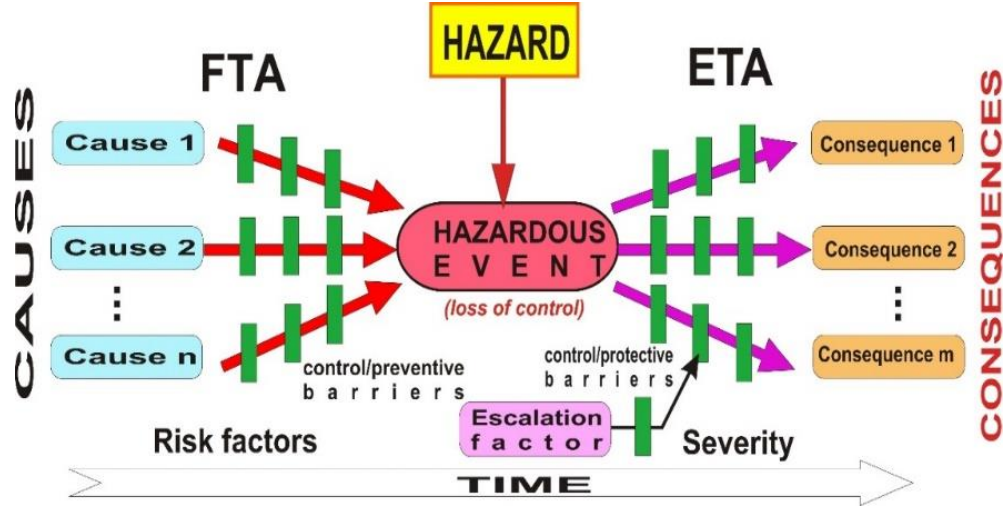

Fig. 1. Typical representation of a Bowtie diagram

A large number of researchers have turned their attention to the Bowtie method. Some of them focused on industry domains such us: mining, chemical and process, oil and gas. Other researchers are turning their attention to the IT, risk management [4, 5], and risk assessment [6-9].

The goals of the Bowtie method are: systematic analysis of a hazard, to help one decide whether the current level of risk control is sufficient, to help one identify the most appropriate places where resources can be allocated so that their efficiency is high.

Improving communication and awareness on risk, causes and effects [2].

On a Bowtie diagram, of the type shown in Fig. 1, are represented the causes that can lead to the occurrence of a dangerous event as well as the possible effects that occur after the occurrence of the dangerous event.

\section{Directions of Analysis in the Bowtie Method}

The Bowtie method incorporates three main directions of analysis: risks, causes and effects [10]:

1. Fault tree analysis - FTA or cause tree analysis is represented on the left side of the diagram and shows how different scenarios can cause loss of control over processes or hazards.

2. Event tree analysis - ETA that is represented by the right side of the diagram which shows the consequences that may occur once control over processes or hazards is lost.

3. Barrier-based thinking, which is based on the Swiss cheese model, a model designed by James Reason in the early '90s.

The "Swiss cheese" model, shown in Figure 2, is a graphical representation of how different barriers or protective layers (LOPA), can fail in the face of a hazard or hazardous situation.

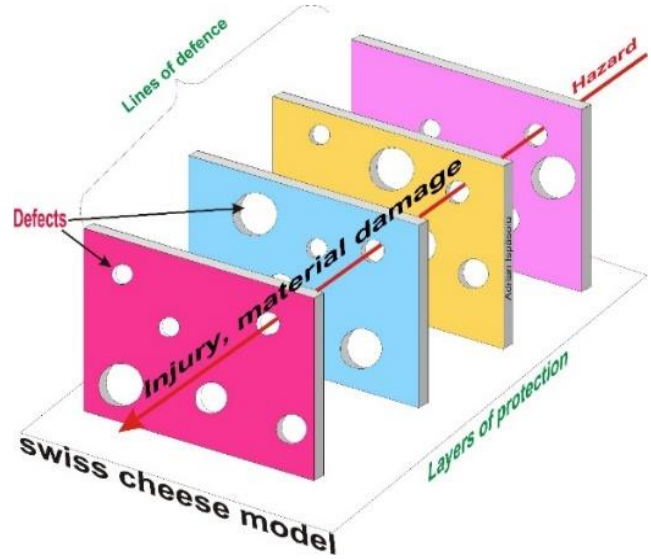

Fig. 2. Swiss cheese model

The Bowtie diagram can also be used to conduct a LOPA study (Layer of Protection Analysis), a simple risk assessment tool to determine if existing protections are sufficient and to calculate the residual risk if they are insufficient [11]. 


\section{The Directions of Analysis in Practice of the Bowtie Method}

The application of the Bowtie method in practice is done in stages.

It starts with identifying the hazard. A hazard can be a source, a situation, or an action with the potential to cause injury or damage. When control of the hazard is lost then the hazardous event or top event (TE) occurs. Loss of control means that one or more protective barriers have failed to perform their function. The causes or security breaches are those that make the occurrence of the hazardous event possible. The consequences are the effects that result from the escalation of the hazardous event in time. Risk management is the manner in which we control risks. The risk can be controlled by placing barriers or controls such as: equipment, processes, workers, etc., on both sides of the hazardous event.

The barriers that exist between the cause and the hazardous event are called proactive barriers. The barriers to the right of the hazardous event have the role of preventing the escalation of this event to the effects caused by the nature of the hazard. These barriers are called reactive barriers because they react to the occurrence of the hazardous event.

The reason for these barrier failures ("holes" in the Swiss cheese model) can often be found within the company. For example, short deadlines for performing an activity may cause workers to stop using appropriate and safe work equipment in order to perform the tasks, but to resort to improvisations or other unsafe methods (Fig. 3), which can lead to musculoskeletal disorders or even to more serious injuries.

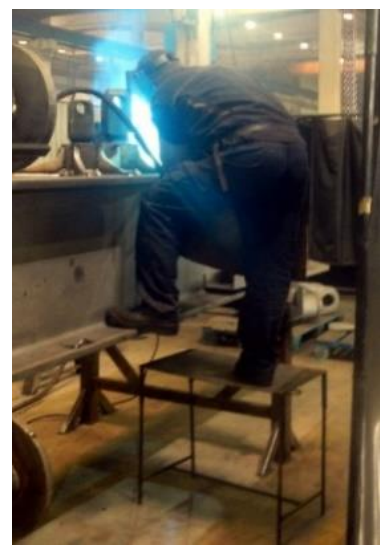

Fig. 3. Unsafe work situation

Analyzing Figure 3, the uncomfortable posture of the worker can be noticed, which can cause musculoskeletal disorders. The use of improvisations can lead to serious accidents.

In the Bowtie method, these deviations from security can be represented as escalating factors. The representation of escalation factors is very important because it allows us to obtain information on the specific conditions under which barriers are affected, removed or overcome. Barriers are never perfect. Even the technical ones, no matter how well built and located, can fail to perform their safety function. For this reason, we need to know how a barrier can fail and what effects this can have on the safety of workers and goods. And the Bowtie method also allows the analysis of how a barrier can fail to function properly, that is, highlighting and describing escalating factors.

Bowtie analysis provides the opportunity to assess the probability and severity of risks, as well as to document the causes of risks, assign and monitor control of risks, and to systematically assess the full range of factors that contribute to workers' exposure to these risks [3].

\subsection{BTRA method parameters}

In this paper, a semi-quantitative method (BTRA - Bowtie Risk Assessment) of estimating and evaluating the ergonomic risk was used, the method having the results of the Bowtie diagram as input data [12].

In this evaluation method were used the following terms: Harm - physical injury or damage to health [12]; Hazard - potential source of harm [12]; Hazardous event (Top Event) - the moment when control 
over the hazard/risk is lost; Severity (G) - determines the nature and type of impact that could occur assuming that a particular event, situation or circumstance has occurred; Probability (P) - probability that the injury will occur; Risk (R) - combination of the probability of occurrence of harm and the severity of that harm [12]; Efficiency (E) - it is an assessment of how effective prevention and protection measures are in controlling risk; Critical, Criticality (C) - represents the influence of the analyzed factor on safety, security and health; Escalation Factor (FE) - are those factors that act on barriers and prevent them from performing the function of safety or protection.

Risk (R) is a combination of SEVERITY (G) and PROBABILITY (P), usually $\mathrm{R}=\mathrm{G} \times \mathrm{P}$.

A risk matrix, presented in Table 1, is used to estimate and assess the risk.

Table 1. Risk matrix - Adapted from Bowtie Methodology, BowTie Pro [1]

\begin{tabular}{|c|c|c|c|c|c|c|c|c|c|}
\hline \multirow{3}{*}{ 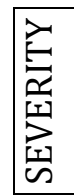 } & \multicolumn{4}{|c|}{ CONSEQUENCES } & \multicolumn{5}{|c|}{ PROBABILITY } \\
\hline & \multirow[b]{2}{*}{$\begin{array}{l}\text { People } \\
\text { (injury) }\end{array}$} & \multirow[b]{2}{*}{$\begin{array}{c}\text { Assets } \\
\text { (damage) }\end{array}$} & \multirow[b]{2}{*}{$\begin{array}{c}\text { Environment } \\
\text { (effect) }\end{array}$} & \multirow[b]{2}{*}{$\begin{array}{c}\text { Reputation } \\
\text { (impact) }\end{array}$} & 1 & 2 & 3 & 4 & 5 \\
\hline & & & & & Unlikely & Unusual & Possible & Likely & $\begin{array}{l}\text { Almost } \\
\text { certain }\end{array}$ \\
\hline 1 & $\begin{array}{l}\text { Minor or } \\
\text { not at all }\end{array}$ & $\begin{array}{l}\text { Minor or } \\
\text { not at all }\end{array}$ & $\begin{array}{l}\text { Minor or } \\
\text { not at all }\end{array}$ & $\begin{array}{l}\text { Minor or } \\
\text { not at all }\end{array}$ & Small & Small & Small & Medium & Medium \\
\hline 2 & Minor & Minor & Minor & Limited & Small & Medium & Medium & Medium & High \\
\hline 3 & Major & Local & Local & Major & Small & Medium & Medium & High & High \\
\hline 4 & Death & Major & Major & National & Medium & Medium & High & High & High \\
\hline 5 & Deaths & Extended & Massive & International & Medium & High & High & High & High \\
\hline
\end{tabular}

The matrix represented in Table 1, consists of the representation of the consequences on people, assets, environment, reputation, on a scale of severity with values from 1 to 5 and on a scale of the probability of injury / damage with values from 1 to 5 . In this matrix, the level of risk is represented on 3 areas: small, medium, high.

A symmetric matrix was used to represent the level of risk. The combination Consequence $=5$ (or Consequence $=4$ ) and Probability = 1 was chosen as Medium risk level because there are risks that can have the maximum Consequence of death or even more deaths (example: explosion) but that are well kept under control. If the level of risk had been chosen as High for these risks, then all the risks that would have had as maximum Severity death, would be unacceptable and, in order to become acceptable, the measures would have had to intervene on the nature of the danger so that the value of Severity is reduced, but, in most situations, this technically impossible or very expensive.

The combination of Consequence $=4$ and Probability $=2$ was chosen as Medium risk level because there are risks that may have the Consequence of death but which are well controlled by the risk owner but it may also happen that a component of the Probability of injury cannot be fully controlled at all times. For example, inappropriate human behavior (human error) or other causes that are independent of the employer's control increase the probability of injury.

The description of the significance of the risk areas is presented in Table 2.

Table 2. Significance of risk areas

\begin{tabular}{|c|l|l|}
\hline Risk level & \multicolumn{2}{|c|}{ Description } \\
\hline $1-3$ & Small & Tolerable \\
\hline $4-9$ & Medium & ALARP (Tolerable) \\
\hline $10-25$ & High & Intolerable \\
\hline
\end{tabular}

When the estimated values for Severity and Probability are low and the risk levels resulting from the value of the level of Seriousness multiplied by the level of Probability are less than or equal to 3, the risks are considered low. Risk levels in the range $4-9$ are considered medium level and classified as ALARP (as low as reasonably practicable). These risks are tolerable but must be constantly monitored, as they may become intolerable (namely loss of safety and occurrence of injury) at the slightest deviation from safety rules. Risks with levels above 10 are high risks that cannot be tolerated and 
assumed. Of course, depending on the particular security criteria of an enterprise, these scales may be changed accordingly by the assessment team before the risk assessment begins.

The efficiency of the controls and the criticality of the factors are described in Table 3. Estimating the effectiveness of controls is a particularly important step in risk assessment, as the safety of workers depends on how those controls control hazards/risks. The probability of material injury or damage is directly proportional to the effectiveness of the controls.

Table 3. Efficiency of controls and criticality of factor

\begin{tabular}{|l|l|l|l|}
\hline \multicolumn{2}{|c|}{ EFFIENCY } & \multicolumn{2}{c|}{ Critical Factor } \\
\hline 5 & Ineffective & 1 & No \\
\hline 4 & Small & 2 & Yes \\
\hline 3 & Average & & \\
\hline 2 & High & & \\
\hline 1 & Very high & & \\
\hline
\end{tabular}

The formula for calculating the probability (Pc) that a particular cause will lead to the Hazardous Event (Top Event) is presented in relation (1):

$$
P c=E \times C
$$

The formula for calculating the Probability (P) for the Hazardous Event (Top Event) to occur, taking into account all identified causes, is presented in relation (2):

$$
\mathrm{Pt}=\frac{\sum_{\mathrm{i}=1}^{\mathrm{n}} \mathrm{E}_{\mathrm{i}} \cdot C i}{n}=\frac{\sum_{\mathrm{i}=1}^{\mathrm{n}} \mathrm{Pc}_{\mathrm{i}}}{n}
$$

where: Pt - the probability that the Top Event will occur; $\mathrm{n}$ - the number of identified causes; $\mathrm{E}$ - the effectiveness of controls for a cause/threat; C - criticality of the cause; Pc - the probability that a specific cause will lead to the occurrence of the Top Event.

Correspondence for the calculation of the probability between the values obtained in the calculation and the scale from 1 to 5 is presented in Table 4.

Table 4. Correspondence for calculating the probability and severity

\begin{tabular}{|l|l|c|c|c|c|c|c|c|c|c|}
\hline Probability & 1 & 2 & 3 & 4 & 5 & 6 & 8 & 10 & Severity \\
\hline 1 & Unlikely & $\mathrm{X}$ & $\mathrm{X}$ & & & & & & & 1 \\
\hline 2 & Rare & & & $\mathrm{X}$ & $\mathrm{X}$ & & & & & 2 \\
\hline 3 & Possible & & & & & $\mathrm{X}$ & $\mathrm{X}$ & & & 3 \\
\hline 4 & Probable & & & & & & & $\mathrm{X}$ & & 4 \\
\hline 5 & Almost certain & & & & & & & & $\mathrm{X}$ & 5 \\
\hline
\end{tabular}

The formula for calculating the Severity (Gc) of a certain consequence is presented in relation (3):

$$
G c=E \times C
$$

The Severity (Gt) calculation formula, taking into account all causes is shown in relation (4):

$$
\mathrm{Gt}=\frac{\sum_{\mathrm{i}=1}^{\mathrm{n}} \mathrm{E}_{\mathrm{i}} \cdot C i}{n}=\frac{\sum_{\mathrm{i}=1}^{\mathrm{n}} \mathrm{Gc}_{\mathrm{i}}}{n}
$$

where: Gt - the severity that the hazard generates after the Top Event has occurred; $\mathrm{n}$ - number of consequences identified; $\mathrm{E}$ - the effectiveness of controls to prevent a certain consequence from occurring; C - criticality of the consequence; Gc - the severity that a certain consequence may have after the occurrence of the Top Event.

The correspondence table to reduce the severity level on a scale from 1 to 5 is presented in Table 4 .

The total level of risk that the Hazardous Event may have is calculated by the formula in relation (5): 


$$
G=G t \times P t
$$

\section{Experimental Results and Discussions}

Analyzing the situation presented in Figure 3, it was build the Bowtie diagram for the activity: welding of metal parts, activity associated with "hazard" because it generates many risks of injury and damage to workers' health, and it was considered the hazardous event as the incorrect positions of the worker, as can be seen in Figure 3. To build the Bowtie diagram, was used the BowTie XP software application, version 10.0.4.0., Trial version, belonging to CGE Risk Management Solution.

The left side of the Bowtie diagram, in Figure 4, highlights the cause tree (FTA) that can lead to the hazardous event. The right side of the diagram represents the possibilities for the events to be carried out (ETA) after the hazardous event takes place.

Analyzing these data from the Bowtie diagram (Fig. 4), the ergonomic risk assessment was performed, for the dangerous event analyzed, applying the BTRA method, and was obtained the risk analysis represented in Table 6. In this assessment only the Severity on persons will be analyzed.

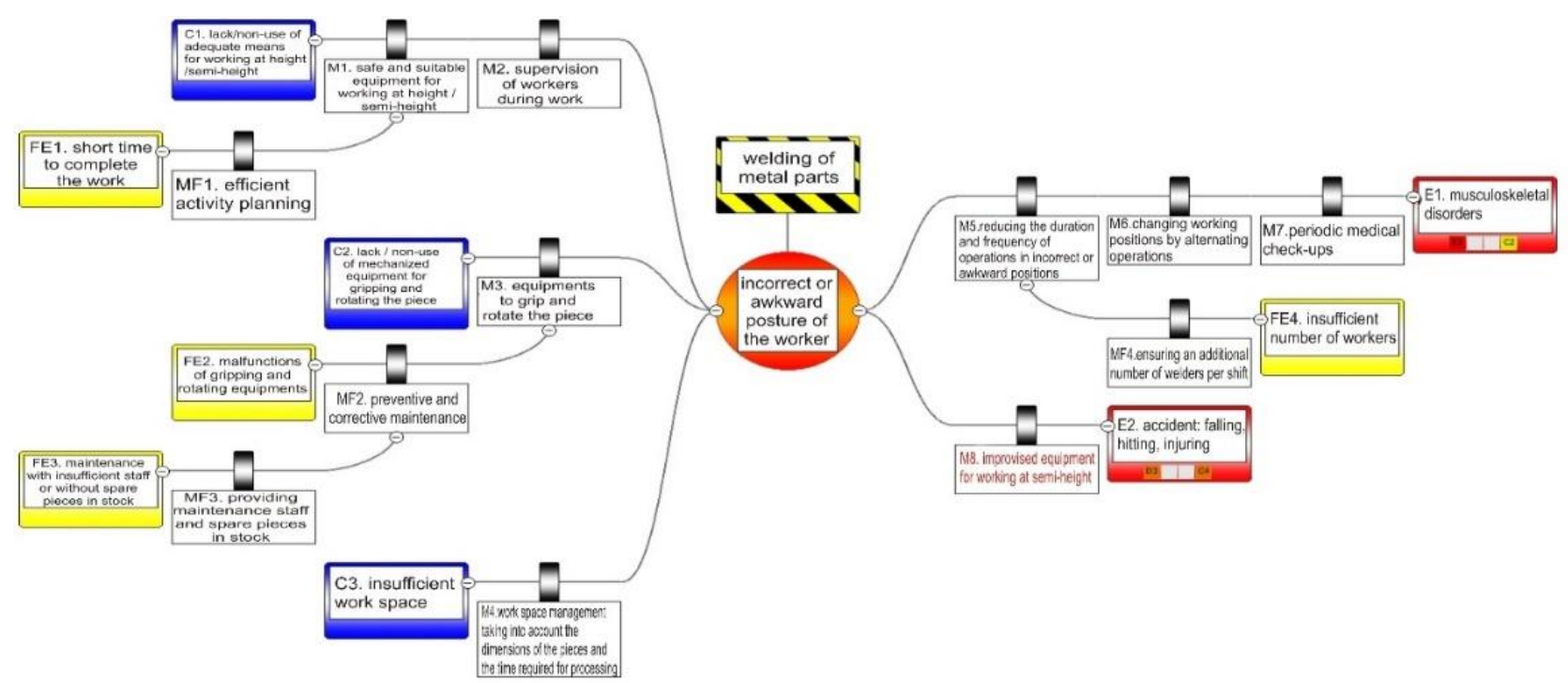

Fig. 4. The complete Bowtie diagram

The header of the risk analysis and assessment is represented in Table 5. It is called the danger or activity, the top event (the dangerous event) and the description of the work situation that must be analyzed and evaluated.

Table 5. Risk analysis and assessment

Hazard/Activity: Welding of metal pieces

Top Event: Incorrect or awkward position of the worker

Description: The workpiece is positioned on a fixed device. The worker welds at a height of approx. 180 $\mathrm{cm}$ from the floor. The worker is mounted on an improvised device. The position of the worker is: the body bent and twisted; the legs are not at the same level. Arms raised and half-stretched. Duration: 5-10 minutes/position. Frequency: about 20 /turn.

Table 6 identifies and analyzes the causes and proactive measures as well as their efficiency and criticality, then calculates the probability of occurrence of the dangerous event.

Table 7 identifies and analyzes the possible consequences and reactive measures to the dangerous event, as well as their efficiency and criticality, then calculates the severity for each consequence (for example consequence E1) on the objective.

The Table 8 shows the estimation and evaluation of the risk level for each consequence (for example consequence E1). 
RECENT, Vol. 22, no. 3(65), 2021

Table 6. Analysis of causes and proactive measures - those that influence Probability

\begin{tabular}{|c|c|c|c|c|c|c|}
\hline Cause & Barriers & $\mathrm{FE}$ & FE control & $(\mathrm{E})^{*}$ & & $\mathrm{Pc}$ \\
\hline \multirow{2}{*}{$\begin{array}{c}\text { C1. } \\
\text { Lack/non-use } \\
\text { of adequate } \\
\text { means for } \\
\text { working at } \\
\text { height/semi- } \\
\text { height }\end{array}$} & $\begin{array}{c}\text { M1. } \\
\text { Safe and suitable means } \\
\text { for working at } \\
\text { height/semi-height }\end{array}$ & $\begin{array}{l}\text { FE1. } \\
\text { The short time assigned } \\
\text { to complete the work }\end{array}$ & $\begin{array}{c}\text { MF1. } \\
\text { Effective business } \\
\text { planning }\end{array}$ & \multirow[t]{2}{*}{2} & \multirow[t]{2}{*}{2} & \multirow{2}{*}{$\begin{array}{c}4 \\
\text { (Rare) }\end{array}$} \\
\hline & $\begin{array}{c}\text { M2. } \\
\text { Supervision of workers } \\
\text { during work }\end{array}$ & & & & & \\
\hline \multirow{2}{*}{$\begin{array}{l}\text { C2. } \\
\text { Lack / non-use } \\
\text { of mechanized } \\
\text { means of } \\
\text { gripping and } \\
\text { rotating the } \\
\text { workpiece }\end{array}$} & $\begin{array}{l}\text { M3. } \\
\text { Devices that grip and } \\
\text { rotate the workpiece }\end{array}$ & $\begin{array}{l}\text { FE2. } \\
\text { Defective gripping and } \\
\text { rotating means }\end{array}$ & $\begin{array}{c}\text { MF2. } \\
\text { Preventive and } \\
\text { corrective } \\
\text { maintenance }\end{array}$ & \multirow[b]{2}{*}{3} & \multirow[b]{2}{*}{2} & \multirow[b]{2}{*}{$\begin{array}{c}6 \\
\text { (Possible) }\end{array}$} \\
\hline & & $\begin{array}{c}\text { FE3. } \\
\text { Maintenance with } \\
\text { insufficient staff or } \\
\text { without spare parts in } \\
\text { stock }\end{array}$ & $\begin{array}{c}\text { MF3. } \\
\text { Providing the } \\
\text { necessary staff } \\
\text { and stocks of } \\
\text { spare parts }\end{array}$ & & & \\
\hline $\begin{array}{c}\text { C3. } \\
\text { Insufficient } \\
\text { workspace }\end{array}$ & $\begin{array}{l}\text { M4. } \\
\text { Workspace management } \\
\text { taking into account the } \\
\text { dimensions of the } \\
\text { workpieces and the time } \\
\text { required for } \\
\text { processing }\end{array}$ & & & 4 & 1 & $\begin{array}{c}4 \\
\text { (Rare) }\end{array}$ \\
\hline \multicolumn{6}{|c|}{ otal Pc } & 4.67 \\
\hline
\end{tabular}

Table 7. Analysis of consequences and reactive measures-those that can influence Severity (E1)

\begin{tabular}{|c|c|c|c|c|c|c|}
\hline Consequences & Existing measures & $\mathrm{FE}$ & FE control & $(E)^{*}$ & (C)* & Gc \\
\hline \multirow{3}{*}{$\begin{array}{c}\text { E1. } \\
\text { Musculoskeletal } \\
\text { disorders }\end{array}$} & $\begin{array}{l}\text { M5. } \\
\text { Reducing the duration and } \\
\text { frequency of operations in } \\
\text { incorrect or uncomfortable } \\
\text { positions } \\
\text { p }\end{array}$ & $\begin{array}{c}\text { FE4. } \\
\text { Insufficient } \\
\text { number of } \\
\text { workers }\end{array}$ & $\begin{array}{c}\text { MF4. } \\
\text { Providing an } \\
\text { additional number } \\
\text { of welders per shift }\end{array}$ & \multirow{3}{*}{3} & \multirow{3}{*}{2} & \multirow{3}{*}{6} \\
\hline & $\begin{array}{c}\text { M6. } \\
\text { Changing working positions by } \\
\text { alternating operations }\end{array}$ & & & & & \\
\hline & $\begin{array}{c}\text { M7. } \\
\text { Periodic medical check-up }\end{array}$ & & & & & \\
\hline
\end{tabular}

Table 8. Calculation of the risk level for the consequence E1

\begin{tabular}{|l|c|l|}
\hline \multicolumn{3}{|l|}{ Calculation of the risk level for the consequence E1 } \\
\hline Severity (personal injury) (G) & 3 & Major effects \\
\hline Probability (P) & 3 & Possible \\
\hline Risk level (R) & & Medium \\
\hline
\end{tabular}

The Table 9 shows the analysis of consequences and measures-those that can influence Severity. The Table 10 shows the estimation and assessment of the risk level for the consequence E2. The Table 9 and Table 10 shows the same type of analysis as in Tables 7, but for the consequence E2. 
RECENT, Vol. 22, no. 3(65), 2021

Table 9. Analysis of consequences and reactive measures-those that can influence Severity (E2)

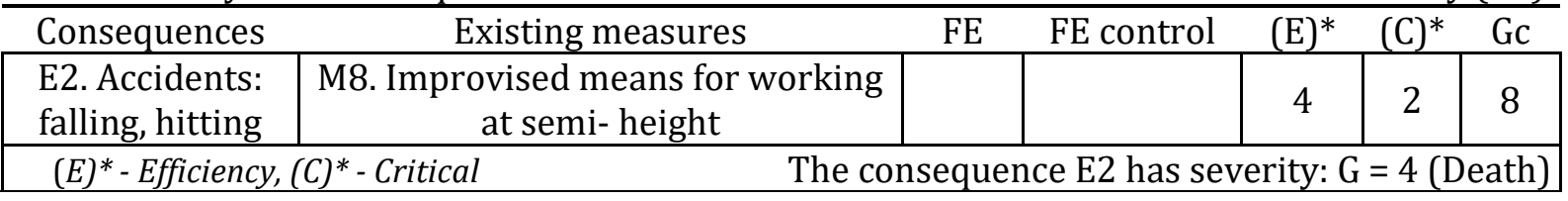

Table 10. Estimation and assessment of the risk level for the consequence E2

\begin{tabular}{|c|c|c|}
\hline \multicolumn{3}{|c|}{ Calculation of the risk level for the consequence E2 } \\
\hline Severity (personal injury) (G) & 4 & Major effects \\
\hline Probability (P) & 3 & Possible \\
\hline \multicolumn{2}{|c|}{ Risk level (R) } & High \\
\hline
\end{tabular}

The debates on the results obtained and the effectiveness of the BTRA method show that the assessment of the risks related to ergonomics in a "classical" manner, highlights both the way in which the health of workers is affected due to awkward and insecure positions and the way in which problems related to ergonomics (e.g. awkward positions) affect the safety of workers, namely they can cause serious accidents.

The BTRA method estimates the level of risk and assesses the risk for each consequence. These consequences are identified and showed in the Bowtie diagram. It can be seen that the risk assessment resulting directly from the Bowtie diagram is systematic and with a high degree of rigor, qualities observable even in the way in which the risks can be defined, for example: "risk of falling from a height / semi-height of the worker standing in an incorrect working position, a position caused by the lack /non-use of adequate means for working at height". Thus, a risk expressed in this way can be treated specifically as compared to a risk with the same effect but with a different cause and therefore possibly a different level of risk, because there may be another probability of occurrence of that risk at the same severity level.

Analyzing the results of the assessment of the ergonomic risks generated by incorrect and/or uncomfortable positions during the activity of welding of the workpieces in the production hall, it is found that there is a high probability of occurrence of musculoskeletal disorders but also of serious injury to the worker caused in particular by the non-use of the appropriate means of working at height/semi-height and the use of improvisations, as well as due to the lack of mechanized means for gripping and rotating the workpiece in a position favorable to the worker.

This study has some limitations. The impact of applying the BTRA method in other fields of activity has not been studied. The results of the analyzed example cannot be implicitly generalized.

\section{Conclusions}

Analyzing the results obtained in this paper, it can be concluded the following:

1. The Bowtie methodology has proven its efficiency and effectiveness in identifying causes and effects in the event of occurrence of a Hazardous Event (Top Event). Visualizing the causes, consequences, barriers and escalating factors facilitates the identification of possible vulnerabilities and the taking of necessary measures so that the hazardous event does not occur.

2. The Bowtie method can play an important role in improving job security programmers.

3. The BTRA assessment method is also easy to use in combination with the Bowtie method, taking into account the causes and consequences and assessing the effectiveness of proactive and reactive barriers, then classifying the criticality of causes and effects and finally estimating the probability and severity values based on which the risk is assessed.

\section{References}

1. Alizadeh S.S., Moshashaei P. (2015): The Bowtie method in safety management system: A literature review. Scientific Journal of Review, ISSN 2322-2433, Vol. 4, no. 9, pp. 133-138, doi: 10.14196/sjr.v4i9.1933

2. Gerkensmeier B., Ratter B.M.W. (2018): Multi-risk, multi-scale and multi-stakeholder-the contribution of a bowtie analysis for risk management in the trilateral Wadden Sea Region. Journal of Coastal Conservation, ISSN 1874-7841, Vol. 22, no. 1, pp. 145-156, doi:10.1007/s11852-016-0454-8 
3. The Bowtie Methodology. BowTie Pro, http://downloads.bowtiepro.com/es-BowtieMethodology.pdf

4. Cockshott J.E. (2005): Probability bow-ties: a transparent risk management tool. Process Safety and Environmental Protection, ISSN 0957-5820, Vol. 83, no. 4, pp. 307-316, https://doi.org/10.1205/psep.04380

5. Mokhtari K., Ren J., Roberts C., Wang J. (2011): Application of a generic bow-tie based risk analysis framework on risk management of sea ports and offshore terminals. Journal of Hazardous Materials, ISSN 0304-3894, Vol. 192, no. 2, pp. 465-475, https://doi.org/10.1016/i.jhazmat.2011.05.035

6. Aqlan F., Mustafa Ali, E. (2014): Integrating lean principles and fuzzy bow-tie analysis for risk assessment in chemical industry. Journal of Loss Prevention in the Process Industries, ISSN 0950-4230, Vol. 29, no. 1, pp. 39-48, https://doi.org/10.1016/i.jlp.2014.01.006

7. Jacinto C., Silva C. (2010): A semi-quantitative assessment of occupational risks using bow-tie representation. Safety Science, ISSN 0925-7535, Vol. 48, No. 8, pp. 973-979, https://doi.org/10.1016/i.ssci.2009.08.008

8. Chevreau F.R., Wybo J.L., Cauchois D. (2006): Organizing learning processes on risks by using the bow-tie representation. Journal of Hazard Material, ISSN 1873-3336, Vol. 130, no. 3, pp. 276-283, https://doi.org/10.1016/j.jhazmat.2005.07.018

9. Ferdous R., Khan F., Sadiq R., Amyotte P., Veitch B. (2012): Handling and updating uncertain information in bowtie analysis. Journal of Loss Prevention in the Process Industries, ISSN 1873-3352, Vol. 25, no. 11, pp. 8-19, https://doi.org/10.1016/j.jlp.2011.06.018

10. The Bowtie method. CGE Risk, https://www.cgerisk.com/knowledgebase/The bowtie method

11. Levis S., Smith K. (2010): Lessons Learned from Real World Application of the Bow-tie Method. $6^{\text {th }}$ Global congress on process safety (GCPS 2010), https://citeseerx.ist.psu.edu/viewdoc/download?doi=10.1.1.466. 3566\&rep=rep1\&type=pdf

12. EN ISO 12100:2010 Safety of machinery - General principles for design - Risk assessment and risk reduction. https://www.iso.org/standard/51528.html 